\title{
Ultrasound guided axillary brachial plexus block
}

\author{
Anil Ranganath, Karthikeyan Kallidaikurichi Srinivasan, Gabriella Iohom
}

Department of Anaesthesia and Intensive Care Medicine, Cork University Hospital and University College Cork, Wilton, Cork, Ireland

\begin{abstract}
The axillary brachial plexus block is the most widely performed upper limb block. It is relatively simple to perform and one of the safest approaches to brachial plexus block. With the advent of ultrasound technology, there is a marked improvement in the success rate of the axillary block. This review will focus on the technique of ultrasound guided axillary brachial plexus block.
\end{abstract}

Keywords: axillary brachial plexus block, regional anaesthesia, ultrasound

\section{Introduction}

Peripheral nerve blocks have seen a big resurgence of interest in the past decade especially with the advent of ultrasound. Nerve blocks have evolved from being an art that only a few physicians can master to more objective and transferable skill largely due to the introduction of ultrasound guidance. Peripheral nerve blocks today are a major component of perioperative multimodal analgesia $[1,2]$. In particular, for upper extremity surgeries, blocks of brachial plexus (interscalene, supraclavicular, infraclavicular and axillary approaches) have been consistently shown to be associated with time-efficient anaesthesia, faster recovery, fewer adverse events, better analgesia, and greater patient acceptance [3-5].

The axillary brachial plexus block (ABPB) provides surgical anaesthesia at and below the elbow. The tech-

Received 10.06.2014 Accepted 26.03.2014

Med Ultrason

2014, Vol. 16, No 3, 246-251

Corresponding author: Gabriella Iohom $\mathrm{MD}, \mathrm{PhD}$

Department of Anaesthesia and Intensive

Care Medicine,

Cork University Hospital and University

College Cork

Wilton Road, Cork, Ireland.

Phone: +35321 4922135

Fax: +353 214546434

E-mail: gabriella.iohom@hse.ie nique is relatively simple to perform because of superficial location and relatively lower risk of complications as compared to interscalene (e.g., phrenic nerve block, spinal cord or vertebral artery puncture) or supraclavicular (e.g., pneumothorax) approaches. Inadvertent intraneural and intravascular injections are the only significant risks. Various methods of ABPB have been described such as paraesthesia-seeking, nerve-stimulating, perivascular, trans-arterial, and ultrasound-guided techniques. This review will focus on the ultrasound guided axillary brachial plexus block.

\section{Anatomy}

The brachial plexus is derived from the ventral rami of C5-8 and T1 nerve roots in most individuals. Occasionally contribution from $\mathrm{C} 4$ and $\mathrm{T} 2$ nerve roots create a 'prefixed' or 'postfixed' plexus [6]. The roots emerge from the intervertebral foramina and continue between scalenus anterior and medius muscles. Here roots unite to form trunks (upper, middle and lower) and pass downward over the posterior neck triangle and the first rib. At the lateral border of the first rib, behind the clavicle, the trunk divides into divisions (anterior and posterior). These divisions continue into the axilla and form the cords. The anterior divisions of the upper and middle trunks unite to form the lateral cord, while the anterior division of the 
lower trunk continues as the medial cord. All three posterior divisions unite to form the posterior cord. These cords are named according to their position around the axillary artery. Each cord ends near the lower border of the pectoralis minor muscle by dividing into two terminal branches. The lateral cord gives off the lateral branch of median nerve and terminates as musculocutaneous nerve. The medial cord gives off the medial branch of median nerve and terminates as ulnar nerve and the posterior cord gives off the axillary nerve and terminates as radial nerve. All these terminal nerves along with the medial cutaneous nerve of arm, forearm and intercostobrachial nerve provide the sensory and motor supply of the upper extremity. The cords, the terminal branches and the vessels lie within an incomplete fascial sheath derived from the prevertebral fascial layer [7].

At the level of axilla, the median, ulnar and radial nerves lie within the neurovascular bundle. The musculocutaneous nerve lies outside the sheath in the plane between the biceps and coracobrachialis muscle, as it leaves the lateral cord before the cords enter the axilla. Within the fascia, in relation to the axillary artery, the nerves are arranged as follows: (1) median-lateral and anterior, (2) ulnar-medial and anterior, and (3) radial-medial and posterior. The musculocutaneous nerve appears lateral and posterior to the artery.

\section{Principles of brachial plexus block}

The brachial plexus can be blocked at its various anatomical divisions from nerve roots to its individual terminal branches. The choice of approach depends upon the sensory and motor innervations of the surgical site. The interscalane approach blocks the plexus at the level of roots, thus it is used for shoulder and proximal humerus procedures. The supraclavicular approach blocks the plexus at the level of trunks and divisions providing the most widespread surgical anaesthesia for the whole arm. The infraclavicular approach blocks the cords, whereas the axillary approach blocks the terminal branches thus providing surgical anaesthesia for the elbow, forearm and hand. In addition, the axillary block also provides cutaneous anaesthesia for the inner upper arm which is suitable for procedures requiring tourniquet. Overall, the axillary approach is considered the safest approach because of the lowest risk of serious complications.

\section{Ultrasound guided axillary brachial plexus block}

Abramowitz and Cohen described in 1981 the use of Doppler ultrasound to identify the axillary artery, an essential landmark during a difficult perform ABPB [8].
It was, however, the use of B-mode ultrasound in 1989 for axillary block performance that paved the way for ultrasound-guided peripheral nerve blocks [9]. Ultrasound guidance is ideally suited for ABPB for a variety of reasons. The nerves are superficial and therefore easier to identify. The shallow depth of the nerve means that the needle for in-plane approach will be almost perpendicular to the direction of the ultrasound beam, thereby greatly improving needle visibility, which in turn will allow for accurate needle positioning with minimal needle redirections. The vast anatomical variations in position of vascular and neural structures relevant to axillary block [10] will make ultrasound even more suited when compared to conventional landmark guided approach. As one would expect, ultrasound guidance has shown to reduce block performance time, improve block success rate [11$13]$, shorten onset time $[14,15]$, reduce vascular puncture $[16,17]$ and achieve a reduction in the volume of local anaesthetic required [18].

\section{Ultrasound anatomy}

The patient is made comfortable in supine position with the arm abducted and the elbow flexed to 90 degrees. After skin and probe preparation, a linear 38-mm, high frequency $10-12 \mathrm{MHz}$ transducer is placed in the transverse plane at the lateral border of pectoralis major muscle to obtain the best view of the brachial plexus. Image quality is optimised with selection of appropriate depth (within 1-2 cm), focus range (within $1 \mathrm{~cm}$ ) and gain. The structures of interest are very superficial with the pulsating axillary artery localised within $1 \mathrm{~cm}$ (fig 1). Easing the pressure on the transducer often reveals one or more axillary veins which is often located medially to the artery (fig 2, fig 3). Surrounding the axillary artery, one will find the three out of four terminal branches of the brachial plexus: the median (superficial and lateral to the artery), the ulnar (superficial and medial to the artery) and the radial (posterior and lateral or medial to the artery) nerves. They often have honey comb appearance with heterogeneous echogenecity. The fourth terminal branch, the musculocutaneous nerve is often seen as a hyperechoic flattened oval shape nerve in the plane between the biceps and coracobrachialis muscles. There is a considerable variation in the position of the nerves among individuals. The median nerve is most commonly seen at 11-12 o'clock position, the ulnar nerve at 2-3 o'clock, the radial nerve at 4-6 o'clock and the musculocutaneous commonly seen at 8-9 at o'clock in relation to the artery [19].

Moving the transducer proximally towards the axilla and distally towards the elbow allows appreciation of the 


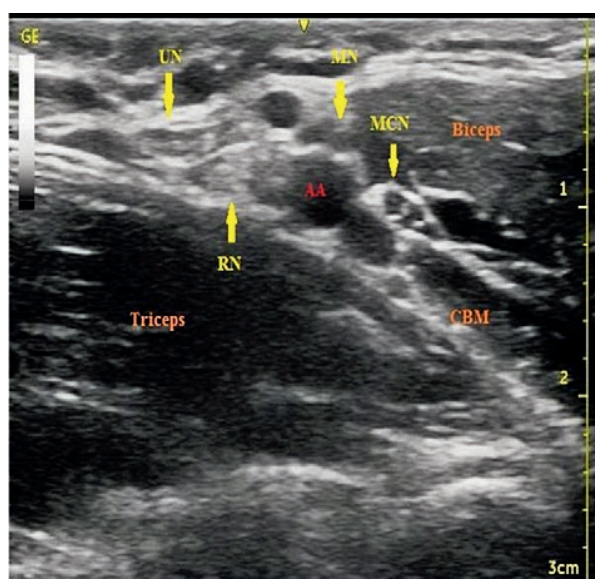

Fig 1. Ultrasound scout scan of axilla showing AA: axillary artery, UN: ulnar nerve, $\mathrm{RN}$ : radial nerve, MCN: musculocutaneous nerve and CBM: coracobrachialis muscle

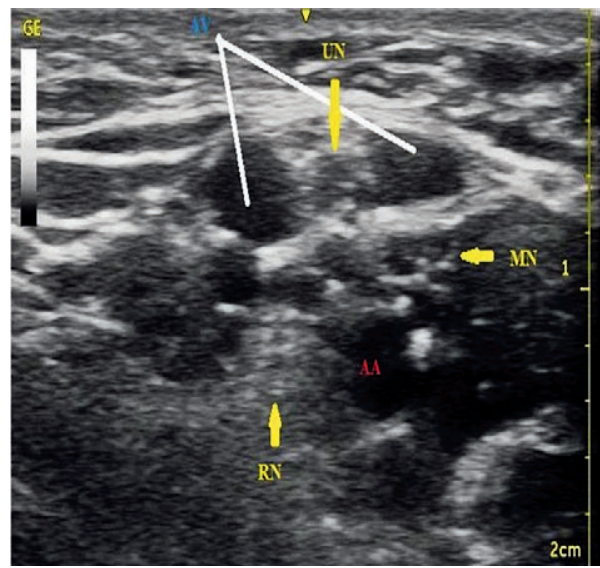

Fig 2. Ultrasound scout scan of axilla showing $\mathrm{AV}$ : axillary vein(s). UN: ulnar nerve, $\mathrm{MN}$ : median nerve, $\mathrm{RN}$ : radial nerve, AA: axillary artery

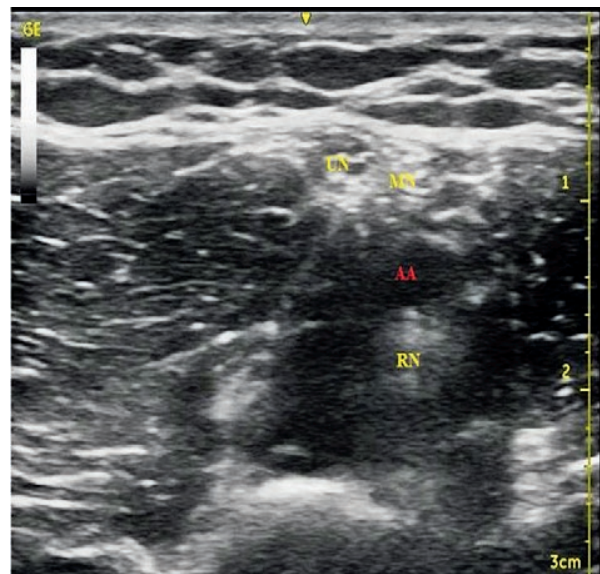

Fig 3. Axillary veins compressed by ultrasound probe

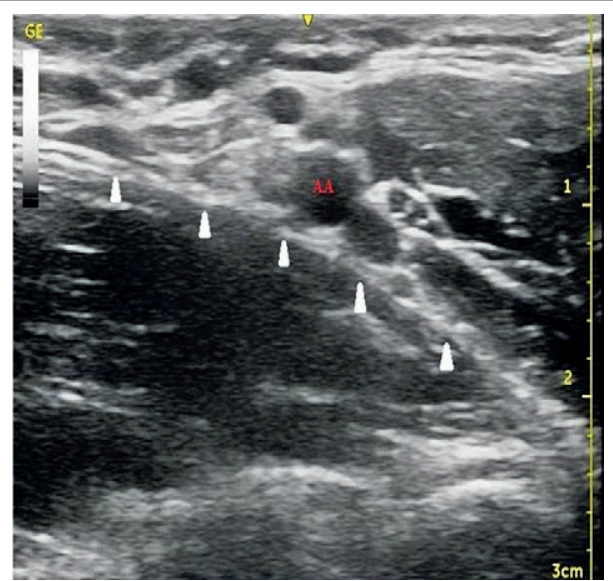

Fig 4. Arrow heads showing sonographic appearance of the conjoint tendon of the latissimus dorsi and teres major muscles

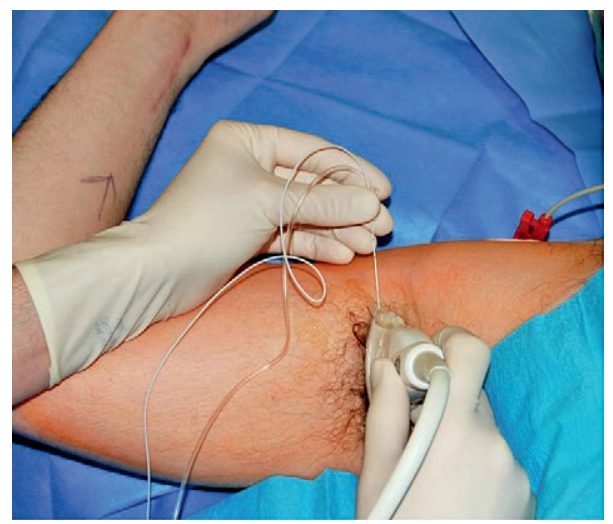

Fig 5. Ultrasound guided axillary brachaial plexus block. In-plane approach of the needle with respect to probe. Also note the position of the patient's upper limb.

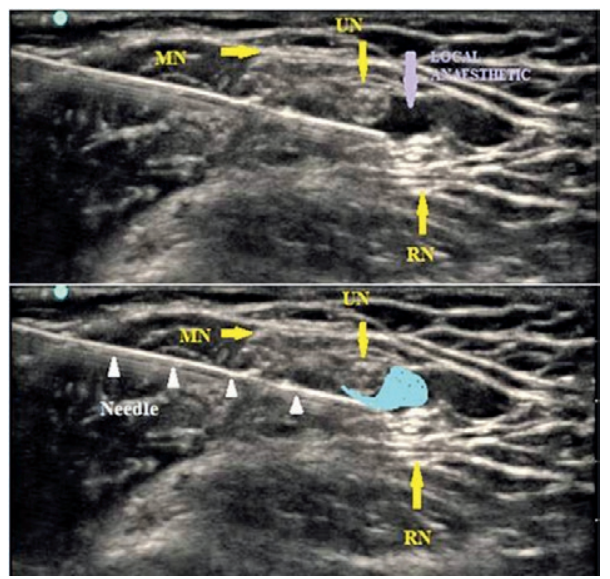

Fig 6. Needle trajectory and local anaesthetic spread around the ulnar nerve (shaded area) 
course of each nerve. Of all the nerve, the radial nerve is often difficult to visualise and block. It is important to exclude the post cystic enhancement artefact beneath the artery. Identification of the confluence of the tendons of the latissimus dorsi and teres major with ultrasound may improve the chance of visualising the radial nerve (fig 4). It lies directly anterior to the humeral insertions of the tendons, with anatomic variation of this relation quite uncommon [20].

\section{Needle insertion}

\section{In plane approach}

A short-bevelled $5 \mathrm{~cm} 22 \mathrm{G}$ insulated needle is inserted parallel to the long axis of the transducer from the lateral side (fig 5). As the needle is in the same plane as an ultrasound beam, the path of the advancement can be visualised in real time as the needle approaches the target nerves (fig 6, fig 7). Ideally, the radial nerve should be targeted first, as it lies posterior to artery, in order to prevent displacing the structures of interest to deeper and obscuring the median and ulnar nerves. The musculocutaneous nerve should be blocked separately outside the neurovascular bundle.

\section{Out of plane approach}

The needle insertion is at the short axis of the probe (fig 8). Constant injection of small quantity of injectate is necessary in order to identify the position of the tip of the needle. In terms of safety, in-plane approach offers better visualisation of the needle.

Importantly, a routine of identifying the axillary veins using colour Doppler (fig 9) as they can be easily compressed with the transducer, and visualising the injectate is paramount. Frequent aspiration, slow administration of 1-2 $\mathrm{mLs}$ of local anaesthetic and visualisation of the spread of injectate around the nerve is critical to reduce the risks of intravascular or intraneural injection and to increase success rate.

\section{Choice and volume of local anaesthetic solution}

This is determined by the desired duration of sensory analgesia. Lidocaine $1.5-2 \%$ or mepivacaine $1-1.5 \%$ with epinephrine 1:200000 provides effective blockade for 2.5-3 hours. A longer duration may be achieved with use of ropivacaine $0.5 \%$ or levobupivacaine $0.5 \%$ [21]. Traditionally, greater volumes of local anaesthetic have been administered to achieve successful axillary brachial plexus block $[22,23]$, but recent studies have demonstrated that this can be achieved with even very low volumes of 2-4 mL lidocaine $1.5 \%$ per nerve [18] or ultra low volume of $1 \mathrm{~mL}$ lidocaine $2 \%$ per nerve [24]. However,

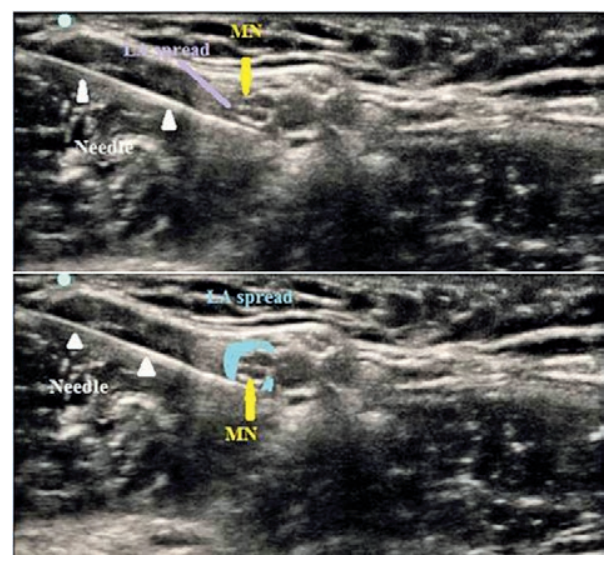

Fig 7. Needle trajectory and local anaesthetic spread around the median nerve (shaded area)

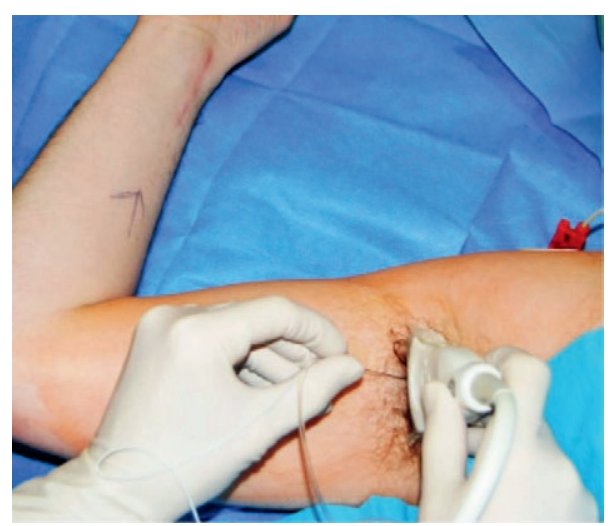

Fig 8. Ultrasound guided axillary brachial plexus block. Out-of-plane approach of the needle with respect to probe.

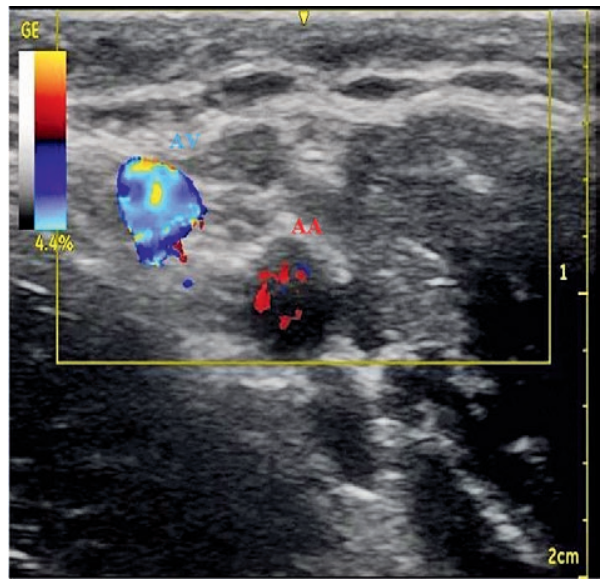

Fig 9. Doppler identification of vessels during ultrasound guided brachial plexus block. 
these volumes were believed to be operator dependent. It is recommended to use at least 4-5 $\mathrm{mL}$ of local anaesthetic solution for each nerve to achieve successful axillary brachial plexus block.

\section{Perivascular vs perineural}

Ultrasound guidance in the performance of axillary block can be applied to

i. perineural injection as described earlier, where the operator identifies and blocks the individual nerves, or

ii. perivascular technique, where the operator blocks the musculocutaneous nerve separately and deposits local anaesthetic solution around the axillary artery, which is in turn believed to achieve blockade of medial, ulnar and radial nerves [25,26].

Both these techniques have been compared by Bernucci et al [27] and found to have similar success rates. However, the latter runs the risk of impaling nerves in inexperienced hands.

\section{Conclusions}

Axillary brachial plexus block is effective and widely used technique for providing surgical anesthesia at and below the elbow. It is relatively simple and safe among the four approaches to brachial plexus. With the advent of ultrasound technology, there is a marked improvement in the success rate, shorter onset time and reduction in the volume required for successful block. Paramount importance should be given to continuous visualisation of the needle advancement, tip position and spread of injectate in order to minimise intravascular and intraneural injection.

\section{Conflict of interest: none}

\section{References}

1. Gritsenko K, Khelemsky Y, Kaye AD, Vadivelu N, Urman RD. Multimodal therapy in perioperative analgesia. Best Pract Res Clin Anaesthesiol 2014; 28: 59-79.

2. Liu SS, Strodtbeck WM, Richman JM, Wu CL. A comparison of regional versus general anesthesia for ambulatory anesthesia: a meta-analysis of randomized controlled trials. Anesth Analg 2005; 101: 1634-1642.

3. Hadzic A, Arliss J, Kerimoglu B, et al. A comparison of infraclavicular nerve block versus general anesthesia for hand and wrist day-case surgeries. Anesthesiology 2004; 101: 127-132.

4. Lehmann LJ, Loosen G, Weiss C, Schmittner MD. Interscalene plexus block versus general anaesthesia for shoulder surgery: a randomized controlled study. Eur J Orthop Surg Traumatol 2014 May 15. DOI 10.1007/s00590-014-14833.

5. O'Donnell BD, Ryan H, O'Sullivan O, Iohom G. Ultrasound-guided axillary brachial plexus block with 20 milliliters local anesthetic mixture versus general anesthesia for upper limb trauma surgery: an observer-blinded, prospective, randomized, controlled trial. Anesth Analg 2009; 109: 279-283.

6. Barash PG. Clinical Anesthesia. 6th ed. Lippincott Williams \& Wilkins; 2009.

7. Barrett HD, Loughnane F, Finucane B, Shorten G. Peripheral nerve blocks and peri-operative pain relief. 1 ed. Philadelphia USA. Saunders; 2004.

8. Abramowitz HB, Cohen C. Use of Doppler for difficult axillary block. Anesthesiology 1981; 55: 603.

9. Ting PL, Sivagnanaratnam V. Ultrasonographic study of the spread of local anaesthetic during axillary brachial plexus block. Br J Anaesth 1989; 63: 326-329.

10. Ustuner E, Yilmaz A, Ozgencil E, Okten F, Turhan SC. Ultrasound anatomy of the brachial plexus nerves in the neurovascular bundle at the axilla in patients undergoing upper-extremity block anesthesia. Skeletal Radiol 2013; 42: 707-713.

11. Chan VW, Perlas A, McCartney CJ, Brull R, Xu D, Abbas $\mathrm{S}$. Ultrasound guidance improves success rate of axillary brachial plexus block. Can J Anaesth 2007; 54: 176-182.

12. Williams SR, Chouinard P, Arcand G, et al. Ultrasound guidance speeds execution and improves the quality of supraclavicular block. Anesth Analg 2003; 97: 1518-1523.

13. Sites BD, Beach ML, Spence BC, et al. Ultrasound guidance improves the success rate of a perivascular axillary plexus block. Acta Anaesthesiol Scand 2006; 50: 678-684.

14. Kapral S, Greher M, Huber G, et al. Ultrasonographic guidance improves the success rate of interscalene brachial plexus blockade. Reg Anesth Pain Med 2008; 33: 253-258.

15. Marhofer P, Schrogendorfer K, Wallner T, Koinig H, Mayer N, Kapral S. Ultrasonographic guidance reduces the amount of local anesthetic for 3-in-1 blocks. Reg Anesth Pain Med 1998; 23: 584-588.

16. Yuan JM, Yang XH, Fu SK, et al. Ultrasound guidance for brachial plexus block decreases the incidence of complete hemi-diaphragmatic paresis or vascular punctures and improves success rate of brachial plexus nerve block compared with peripheral nerve stimulator in adults. Chin Med J (Engl) 2012; 125: 1811-1816.

17. Barrington MJ, Kluger R. Ultrasound guidance reduces the risk of local anesthetic systemic toxicity following peripheral nerve blockade. Reg Anesth Pain Med 2013; 38: 289297.

18. Harper GK, Stafford MA, Hill DA. Minimum volume of local anaesthetic required to surround each of the constituent nerves of the axillary brachial plexus, using ultrasound guidance: a pilot study. Br J Anaesth 2010; 104: 633-636.

19. Christophe JL, Berthier F, Boillot A, et al. Assessment of topographic brachial plexus nerves variations at the axilla using ultrasonography. Br J Anaesth 2009; 103: 606-612. 
20. Gray AT. The conjoint tendon of the latissimus dorsi and teres major: an important landmark for ultrasoundguided axillary block. Reg Anesth Pain Med 2009; 34: 179-180.

21. Gonzalez-Suarez S, Pacheco M, Roige J, Puig MM. Comparative study of ropivacaine $0.5 \%$ and levobupivacaine $0.33 \%$ in axillary brachial plexus block. Re Anesth Pain Med 2009; 34: 414-419.

22. Serradell A, Herrero R, Villanueva JA, Santos JA, Moncho JM, Masdeu J. Comparison of three different volumes of mepivacaine in axillary plexus block using multiple nerve stimulation. Br J Anaesth 2003; 91: 519-524.

23. Vester-Andersen T, Husum B, Lindeburg T, Borrits L, Gothgen I. Perivascular axillary block IV: blockade following 40,50 or $60 \mathrm{ml}$ of mepivacaine $1 \%$ with adrenaline. Acta Anaesthesiol Scand 1984; 28: 99-105.
24. O'Donnell BD, Iohom G. An estimation of the minimum effective anesthetic volume of $2 \%$ lidocaine in ultrasoundguided axillary brachial plexus block. Anesthesiology. 2009; 111: 25-29.

25. Imasogie N, Ganapathy S, Singh S, Armstrong K, Armstrong P. A prospective, randomized, double-blind comparison of ultrasound-guided axillary brachial plexus blocks using 2 versus 4 injections. Anesth Analg 2010; 110: 1222-1226.

26. Tran de QH, Pham K, Dugani S, Finlayson RJ. A prospective, randomized comparison between double-, triple-, and quadruple-injection ultrasound-guided axillary brachial plexus block. Reg Anesth Pain Med 2012; 37: 248-253.

27. Bernucci F, Gonzalez AP, Finlayson RJ, Tran de QH. A prospective, randomized comparison between perivascular and perineural ultrasound-guided axillary brachial plexus block. Reg Anesth Pain Med 2012; 37: 473-477. 